\title{
Active agents of Renin Angiotensin System and SARS-CoV-2
}

\author{
Antonio Vitiello ${ }^{1}$, Raffaele La Porta ${ }^{2}$, Giovanni Granata ${ }^{3}$, Chiara Pelliccia ${ }^{4}$, and Francesco
} Ferrara $^{1}$

${ }^{1}$ Azienda Unità Sanitaria Locale Umbria 1

${ }^{2}$ ASUR Marche

${ }^{3}$ Local Health Authority Salerno

${ }^{4}$ Azienda Unità Sanitaria Locale Umbria 2

May 13, 2020

\begin{abstract}
Introduction The new coronavirus, called SARS-CoV-2, is responsible for the recent outbreak of serious respiratory diseases worldwide. The state of the global pandemic is still being declared and the virus has already claimed thousands of victims. Therapies are urgently needed to contain its rapid spread and reduce high mortality rates, no direct antiviral is yet available and several clinical trials are underway. In addition, no vaccines are currently available and any development in this direction may take several months. Experts in the field have divided SARS-Cov-2 infection into three phases. Materials and methods This article explores the scientific hypothesis based on pharmacological and molecular knowledge to consider drugs that modulate the RAS system as therapeutic agents that can help the body fight SARS-CoV-2 infection. Results It is known from the 2003 SARS epidemic that the critical receptor for SARS-CoV entry into host cells is the angiotensin 2 conversion enzyme (ACE2), the strain involved in the current SARS-CoV-2 epidemic is similar to the SARS-CoV variety involved in the 2002-2003 SARS epidemic. ACE-2 is part of the RAS system, modulating this enzyme could be effective. Conclusions A scientific hypothesis is described, in the absence of studies and clinical data, based on therapeutic treatments that modulate RAS, and current knowledge of the mechanism of penetration of SARS-CoV-2 into cells, and the role of ACE-2 in the inflammatory state of the infection.
\end{abstract}

\section{Active agents of Renin}

\section{Angiotensin System and SARS-CoV-2}

A.Vitiello 1-R.La Porta 2-G.Granata 3-C.Pelliccia 4-F.Ferrara 1*

1:Usl Umbria 1, Perugia, Italy

2:Asur Marche, Ancona, Italy

3:Asl Salerno, Salerno, Italy

4:Usl Umbria 2, Terni, Italy

*author correspondence: francesco.ferrara@uslumbria1.it

\section{Abstract}

\section{Introduction}

The new coronavirus, called SARS-CoV-2, is responsible for the recent outbreak of serious respiratory diseases worldwide. The state of the global pandemic is still being declared and the virus has already claimed thousands of victims. Therapies are urgently needed to contain its rapid spread and reduce high mortality 
rates, no direct antiviral is yet available and several clinical trials are underway. In addition, no vaccines are currently available and any development in this direction may take several months. Experts in the field have divided SARS-Cov-2 infection into three phases.

Materials and methods

This article explores the scientific hypothesis based on pharmacological and molecular knowledge to consider drugs that modulate the RAS system as therapeutic agents that can help the body fight SARS-CoV-2 infection.

ResultsIt is known from the 2003 SARS epidemic that the critical receptor for SARS-CoV entry into host cells is the angiotensin 2 conversion enzyme (ACE2), the strain involved in the current SARS-CoV-2 epidemic is similar to the SARS-CoV variety involved in the 2002-2003 SARS epidemic. ACE-2 is part of the RAS system, modulating this enzyme could be effective. Conclusions A scientific hypothesis is described, in the absence of studies and clinical data, based on therapeutic treatments that modulate RAS, and current knowledge of the mechanism of penetration of SARS-CoV-2 into cells, and the role of ACE-2 in the inflammatory state of the infection.SARS-CoV-2

The SARS-Cov-2 virus spread rapidly in several countries, causing a pandemic with thousands of deaths. SARS-Cov-2 is a family of RNA viruses that can infect humans and promote respiratory tract infections and respiratory distress syndrome. Studies have shown that SARS-Cov-2 has about $80 \%$ similar genome to the SARS-Cov responsible for the 2003 epidemic. Clinical experts and scientists have described SARS-Cov2 infection in three phases, the first asymptomatic or slightly symptomatic, the second moderately severe characterized by a pulmonary inflammatory state, the third very severe phase characterized by a generalized inflammatory state affecting all tissues and not only the lungs. Biochemical interaction studies have shown that SARS-Cov-2 uses the ACE-2 receptor protein to penetrate cells. ACE-2 is also a conversion enzyme that is part of the RAS system. To date, there is as yet no conclusive evidence on the role of the RAS system and ACE-2 in the three stages of SARS-Cov-2 infection and, since they are modulated, we know that there are classes of widely used drugs indicated for cardiovascular diseases that can modulate this system, and that have directly or indirectly a potential positive or negative role in the evolution of the disease. (4-5-6)

\section{The role of ACE-2}

ACE-2 is a type I transmembrane metallocarboxyptidase with ACE homology which is an enzyme known to be a key player in the renin-angiotensin system (RAS) and a target of several drugs for the treatment of high blood pressure. The expression of ACE and ACE-2 is not similar in human tissue. ACE-2 is mainly expressed in vascular endothelial cells, renal tubular epithelium, Leydig cells in the testicles and lung epithelial cells. ACE-2 is a functional receptor of SARS-CoV and SARS-Cov-2 for entry into host cells and subsequent viral replication. Viral entry into respiratory tract cells is a critical step that causes lung injury. Men are thought to be at greater risk of contracting the virus than women because there is a high concentration of ACE-2 in Leydig cells. However, the role of ACE-2 in the pathophysiology of SARS-Cov-2 infection is much more complex than described, since ACE-2 is not only the receptor of the virus entry, in fact the function of ACE-2 in infected lung tissue is currently not very clear, especially in the two most serious stages such as the second and third. where it seems to have a protective role. These new findings could have a great impact on the development of effective therapies for the virus, or to make appropriate use of drugs already on the market that modulate the RAS system to better manage the infection. An interesting line of research is that of anti-ACE-2 antibodies that could be used to block the binding of SARS-CoV-2 to the receptor. (Figure 1). Another possibility that we describe, is the usefulness that a block upstream of the RAS system could have compared to a block downstream at a specific stage of the infection. (7-8-9)

\section{The Renin-Angiotensin System (RAS)}

RAS is a complex cascade event that plays an important role in the control of hydrosaline homeostasis, blood pressure and plasma volume. The cascade begins with renin splitting angiotensinogen, a peptide produced in the liver, into an inactive decapeptide, angiotensin I (Ang I). The latter is converted to the 
active form by another proteolytic enzyme, the angiotensin II conversion enzyme (ACE) produced in the capillary endothelium where it converts angiotensin I (decapeptide) to angiotensin II (Ang II, octapeptide), a powerful vasoconstrictor. ACE, in addition to decoupling Ang I to Ang II, has another important function, it degrades bradykinin into inactive fragments. Bradykinin produces vasodilation through the production of prostaglandins and nitric oxide (NO) and inhibits the proliferation of smooth vascular muscle.

At the cellular level Ang II modulates cell contraction, cell growth, differentiation and apoptosis; it can promote the production of other cytokines, the expression of adhesion molecules and the subsequent recovery of inflammation cells, chemotaxis, macrophage activation. It has a proinflammatory action. The tissue increase of Ang II formation induces inflammation and Ang II is itself a powerful pro-inflammatory cytokine as well as a growth factor. Scientific evidence shows that Ang II activates the transcriptional factor NF-kb, the key factor of nuclear transcription in inflammatory and fibrotic diseases, and its activation allows the transcription of several inflammatory genes, including interleukin 6 and IL-1 which are responsible for the cytokinic cascade and the hyperactive inflammatory state that is generated especially in the third stage of infection. (10-11-12-13)

\section{Active agents on RAS and SARS-CoV-2}

As previously reported SARS-CoV-2 uses the angiotensin 2 conversion enzyme (ACE2) as a receptor binding domain. Based on this, and considering the crucial role of the RAS system and the enzyme ACE-2 in the acute respiratory syndrome caused by SARS-CoV-2 and the correlation with the inflammatory status of lung tissues, it could be considered important to assume that the therapeutic agents acting on RAS, could modulate the viral infection with SARS-CoV-2, with an effect of improvement or worsening, and that managing these drugs in the most timely manner could be of great benefit. The active agents on RAS available may interfere with various stages of the system, an inhibitory action of the enzyme ACE (ACE-i), blocking Angiotensin II (ARB) receptors, inhibitors direct renin. ACE-i and ARB are the drugs of choice for the treatment of hypertension, heart failure and ischemic heart disease.(figure-1)

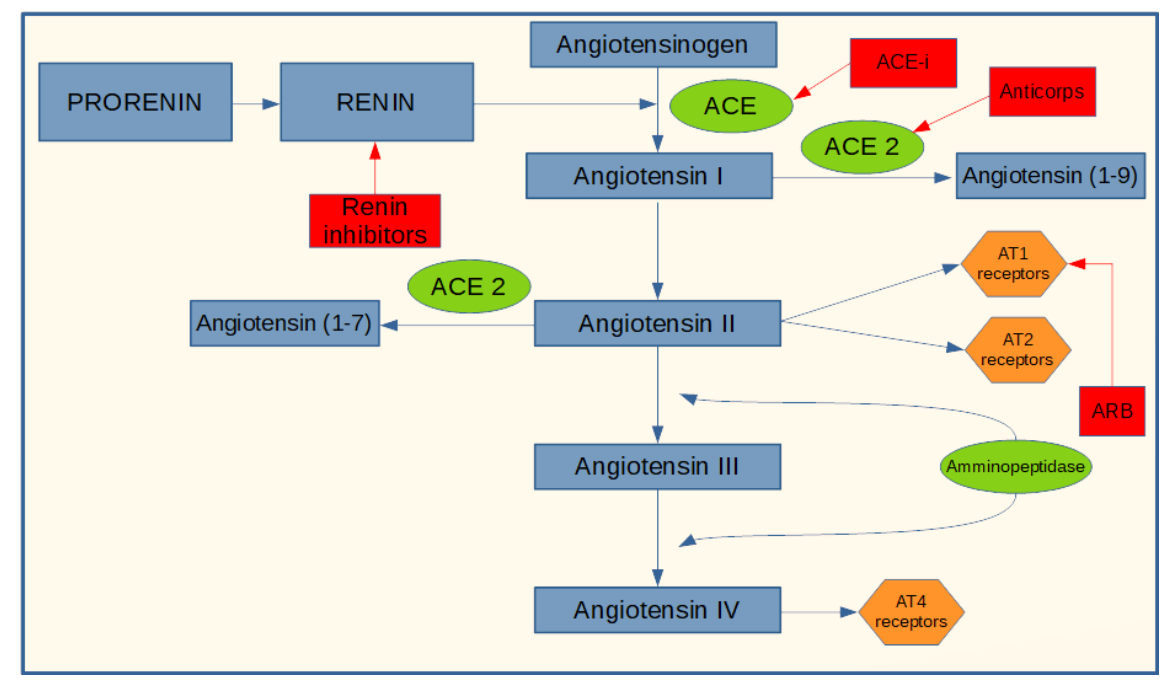

Figure-1: Active agents on the RAS system

These drugs have an extraordinary therapeutic efficacy, but can also cause side effects such as hyperkalaemia, hypotension, cough, angioedema. An increase in ACE-2 concentrations has already been documented both in murine models and in human patients treated with ACE-i and ARB. This is because by inhibiting the ACE pathway, angiotensin I is directed into the conversion pathway to angiotensin I-7, requiring a higher expression of ACE-2 to compensate for the greater amount to be converted. The same mechanism is if you use ARB by blocking the Ang II receptor, in fact the system counter-regulates increasing ACE and ACE-2, in 
addition both ACE-i and ARB cause an increase in the concentration of renin and all the upstream mediators of the enzyme cascade, and this compensatory mechanism may be favorable or unfavorable depending on which stage of the SARS-Cov-2 infection the patient is. With the use of ACE-i or ARB and therefore an increase in the level of expression of the viral receptor mentioned above, an increase in infectious power and colonization could plausibly be obtained. In phase I of the infection, therefore, the virus is penetrating the cell and is replicating, perhaps in this phase it could be useful to administer a direct renin inhibitor which, acting upstream, lowers the concentrations of ACE and especially ACE-2, decreasing the concentration of receptor protein for the virus. On the contrary, in phase two or three of the disease, where there is an hyperactive inflammatory state and where it seems that ACE-2 may have a protective role in particular on the respiratory tract, it may be appropriate to increase ACE-2 with ACE-i or blocking the inflammatory effects of Ang II using ARBs, which also increase the expression of ACE-2 itself. In recent observational studies the most frequent comorbidities reported in patients with SARS-Cov-2 are often treated with (ACE-i); however, the correlation between SARS-Cov-2 and treatment with ACE-i has not been evaluated and demonstrated in any of the studies. Based on PubMed research of 28 February 2020, there is no evidence suggested that direct renin inhibitors cause an increase in ACE2, this drug could therefore be used to manage the hypertension of patients which are in phase I of the infection, or in a preventive (non-virus positive patient) way to reduce the risk of contracting SARS-Cov-2. (14 -15-16-17-18-19-20-21-22-23-24-25-26)

Table 1: Active agents on the RAS system that modulate the concentration of ACE-2 and hypothetical their use in the various stages of SARS-Cov-2 infection.

\section{Conclusion}

In this article we suggest on the basis of today's pharmacological and molecular knowledge, that by modulating the RAS system and the delicate ACE / ACE 2 Ang II balance in the best way and at the right times with common treatments such as ACE-i, ARB and direct renin inhibitors we can have important preventive and curative benefits to fight SARS-Cov-2.

\section{MAIN STATEMENTS}

I, the undersigned, Francesco Ferrara and any other author, declare that:

We have no conflict of interest; We have not received funding; There are no sensitive data and no patients were recruited for this study; The document does not conflict with ethical legislation.

Regards

The authors

\section{References}

1) Baig AM, Khaleeq A, Ali U, Syeda H. Evidence of the COVID-19 Virus Targeting the CNS: Tissue Distribution, Host-Virus Interaction, and Proposed Neurotropic Mechanisms . ACS ChemNeuroscienc. 2020 Mar 13. doi: 10.1021/acschemneuro.0c00122

2) Liu Z, Xiao X, Wei X, Li J, Yang, Tan H, Zhu J, Zhang Q, Wu J, Liu L.JComposition and divergence of coronavirus spike proteins and host ACE2 receptors predict potential intermediate hosts of SARSCoV.MedVirol. 2020 Feb 26. doi: 10.1002/jmv.25726. 2.

3) Cascella M, Rajnik M, Cuomo A, Dulebohn SC, Di Napoli R. Features, Evaluation and Treatment Coronavirus (COVID-19). StatPearls [Internet]. Treasure Island (FL): StatPearls Publishing;2020 Mar 8.

4) Meo SA, Alhowikan AM, Al-Khlaiwi T, Meo IM, Halepoto DM, Iqbal M, Usmani AM, Hajjar W, Ahmed N. Novel coronavirus 2019-nCoV: prevalence, biological and clinical characteristics comparison with SARSCoV and MERS-CoV . Eur Rev Med Pharmacol Sci. 2020 Feb;24(4):2012-2019. 
5) Ashour HM, Elkhatib WF, Rahman MM, Elshabrawy HA. Insights into the Recent 2019 Novel Coronavirus (SARS-CoV-2) in Light of Past Human Coronavirus Outbreaks.Pathogens. 2020 Mar 4;9(3). pi: E186.

6) Walls AC ,Park YJ ,Tortorici MA, Wall A, McGuire AT, Veesler D. Structure, Function, and Antigenicity of the SARS-CoV-2 Spike Glycoprotein.Cell.2020 Mar 6. pii: S0092-8674(20)30262-2.

7) Imai Y, Kuba K, Penninger JM. Angiotensin-convertingenzyme 2 in acute respiratorydistresssyndrome.Cell Mol Life Sci.2007 Aug;64(15):2006-12.

8) Zou X, Chen K, Zou J, Han P, Hao J, Han Z. Single-cell RNA-seq data analysis on the receptor ACE2 expression reveals the potential risk of different human organs vulnerable to 2019-nCoV infection.Front Med. 2020 Mar 12. doi: 10.1007/s11684-020-0754-0. [Epub ahead of print]

9) Batlle D, Wysocki J, Satchell K. Soluble angiotensin-converting enzyme 2: a potential approach for coronavirus infection therapy? Clin Sci (Lond). 2020 Mar 13;134(5):543-545. doi: 10.1042/CS20200163.

10)Tan WSD, Liao W, Zhou S, Mei D, Wong WF. Targeting the renin-angiotensin system as novel therapeutic strategy for pulmonary diseases.CurrOpinPharmacol. 2018 Jun;40:9-17. doi: 10.1016/j.coph.2017.12.002. Epub 2017 Dec 27.

11) Patel S, Rauf A, Khan H, Abu-Izneid T. Renin-angiotensin-aldosterone (RAAS): The ubiquitous system for homeostasis and pathologies.BiomedPharmacother. 2017 Oct;94:317-325. doi: 10.1016/j.biopha.2017.07.091. Epub 2017 Jul 31.

12) Lindgren BR, AnderssonRG.Angiotensin-converting enzyme inhibitors and their influence on inflammation, bronchial reactivity and cough. A researchreview. MedToxicolAdverseDrugExp.1989 Sep-Oct;4(5):36980 .

13) Kim J, Choi SM, Lee J, Park YS, Lee CH, Yim JJ, Yoo CG, Kim YW, Han SK, Lee SM.Effect of Renin-Angiotensin System Blockage in Patients with Acute Respiratory Distress Syndrome:A Retrospective Case Control Study.Korean J Crit Care Med.2017 May;32(2):154-163. doi: 10.4266/kjccm.2016.00976. Epub 2017 May 31.

14) Haibo Zhang, Josef M. Penninger, Yimin Li, Nanshan Zhong\&Arthur S.Angiotensin-converting enzyme 2 (ACE2) as a SARS-CoV-2 receptor: molecular mechanisms and potential therapeutic target. SlutskyIntensive Care Medicine (2020).

15) MirabitoColafella KM, Bovée DM, Danser AHJ.The renin-angiotensin-aldosterone system and its therapeutic targets. Exp Eye Res. 2019 Sep;186:107680. doi: 10.1016/j.exer.2019.05.020. Epub 2019 May 23.

16) Slabý A, Zák A. CasLekCesk.Renin, prorenin, and the direct renin inhibitor aliskiren. 2010;149(2):55-60. Review. Czech.

17) Aulakh GK, Sodhi RK, Singh M.An update on non-peptide angiotensin receptor antagonists and related RAAS modulators. Life Sci. 2007 Aug 2;81(8):615-39. Epub 2007 Jun 28. Review.

18) Ding W, Li X, Wu W,He H, Li Y, Gao L, Gan L, Wang M, Ou S, Liu J. Aliskiren inhibits angiotensin II/angiotensin 1-7(Ang II/Ang1-7) signal pathway in rats with diabetic nephropathy .

19) Yang $\mathrm{X}, \mathrm{Yu} \mathrm{Y}, \mathrm{Xu} \mathrm{J}$, et al. Clinical course and outcomes of critically ill patients with SARS-CoV-2 pneumonia in Wuhan, China: a single-centered, retrospective, observational study. Lancet Respir Med 2020; published online Feb 24.

20) Guan W, Ni Z, Hu Y, et al. Clinical characteristics of coronavirus disease 2019 in China. N Engl J Med 2020; published online Feb 28.

21) Zhang JJ, Dong X, Cao YY, et al. Clinical characteristics of 140 patients infected by SARS-CoV-2 in Wuhan, China. Allergy 2020; published online Feb 19. 
22) Wan Y, Shang J, Graham R, Baric RS, Li F. Receptor recognition by novel coronavirus from Wuhan: An analysis based on decade-long structural studies of SARS . J Virology 2020; published online Jan 29.

23) Li XC, Zhang J, Zhuo JL. Lei Fang, George Karakiulakis, Michael Roth Thevasoprotective axes of the renin-angiotensin system: physiological relevance and therapeutic implications in cardiovascular, hypertensive are patients with hypertension and diabetes mellitus at increased risk for COVID-19 infection? Lancet Respir Med 2020Published OnlineMarch 11, 2020.

24) Andersson RG, Persson K. ACE inhibitors and their influence on inflammation, bronchial reactivity and cough.EurHeart J. 1994 Aug;15 Suppl C:52-6.

25)Gurwitz D. Angiotensin receptor blockers as tentative SARS-CoV-2 therapeutics. Drug Dev Res. 2020 Mar 4. doi: 10.1002/ddr.21656.

26) Drug Saf. 1996 Jul;15(1):72-8.ACE inhibitor-induced cough and bronchospasm. Incidence, mechanisms and management. Overlack A. 